\title{
Preferential Reactivation of Motivationally Relevant Information in the Ventral Striatum
}

\author{
Carien S. Lansink, ${ }^{1}$ Pieter M. Goltstein, ${ }^{1}$ Jan V. Lankelma, ${ }^{1}$ Ruud N. J. M. A. Joosten, ${ }^{1,2}$ Bruce L. McNaughton, ${ }^{3}$ and \\ Cyriel M. A. Pennartz ${ }^{1}$ \\ ${ }^{1}$ Center for Neuroscience, Swammerdam Institute for Life Sciences, University of Amsterdam, 1090 GB Amsterdam, The Netherlands, ${ }^{2}$ Netherlands \\ Institute for Neurosciences, 1105 BA Amsterdam, The Netherlands, and 'Division of Neural Systems, Memory, and Aging, Arizona Research Laboratories, \\ University of Arizona, Tucson, Arizona 85724
}

\begin{abstract}
Spontaneous "off-line" reactivation of neuronal activity patterns may contribute to the consolidation of memory traces. The ventral striatum exhibits reactivation and has been implicated in the processing of motivational information. It is unknown, however, whether reactivating neuronal ensembles specifically recapitulate information relating to rewards that were encountered during wakefulness. We demonstrate a prolonged reactivation in rat ventral striatum during quiet wakefulness and slow-wave but not rapid eye movement sleep. Reactivation of reward-related information processed in this structure was particularly prominent, and this was primarily attributable to spike trains temporally linked to reward sites. It was accounted for by small, strongly correlated subgroups in recorded cell assemblies and can thus be characterized as a sparse phenomenon. Our results indicate that reactivated memory traces may not only comprise feature- and context-specific information but also contain a value component.
\end{abstract}

Key words: nucleus accumbens; learning; memory consolidation; reinforcer; reward; tetrodes

\section{Introduction}

The ventral striatum (VS) is one of the key structures involved in the motivational control of behavior (Mogenson et al., 1980; Robbins and Everitt, 1996). Evidence indicates that the VS is required for the learning of cue-outcome (Parkinson et al., 1999) and response-outcome (Kelley et al., 1997) associations to invigorate or guide goal-directed behavior on the basis of the motivational value of cues and contexts. Single-unit recording studies in awake rodents and primates have established VS neural responsivity to actual and expected rewards or aversive reinforcers (Schultz et al., 1992; Roitman et al., 2005), cues predicting these reinforcers (Tremblay et al., 1998; Setlow et al., 2003; Roitman et al., 2005), and motor responses required to obtain or avoid these (Shidara et al., 1998; Tremblay et al., 1998; Hassani et al., 2001). Recent functional magnetic resonance imaging results have confirmed the role of the VS in processing and predicting reinforcers in a time-specific manner (O’Doherty et al., 2006).

Recently, the VS was indicated to participate in memory consolidation. Pharmacological interventions in protein synthesis,

Received Nov. 1, 2007; revised April 28, 2008; accepted May 12, 2008.

This work was supported by Human Frontier Science Program Grant RGP0127/2001 (C.M.A.P., B.L.M.), Netherlands Organization for Scientific Research-VICI Grant 918.46 .609 and SenterNovem BSIK Grant 03053 (C.M.A.P.), and National Institute of Mental Health Grant MH046823 (B.L.M.). We thank Jolanda Verheul for help with data analysis; A. D. Redish and P. Lipa for the use of cluster-cutting software MClust and BBClust, respectively; and our colleagues at the technology departments of University of Amsterdam and Netherlands Institute for Neurosciences. The comments on this manuscript by Francesco Battaglia, Jadin Jackson, and Sander Daselaar are greatly acknowledged.

Correspondence should be addressed to Cyriel M. A. Pennartz, Center for Neuroscience, Swammerdam Institute for Life Sciences, University of Amsterdam, P.0. Box 94084, 1090 GB Amsterdam, The Netherlands. E-mail: pennartz@science.uva.nl.

DOI:10.1523/JNEUROSCI.1054-08.2008

Copyright $\odot 2008$ Society for Neuroscience $\quad$ 0270-6474/08/286372-11\$15.00/0 glutamatergic or dopaminergic neurotransmission in the VS shortly after training impaired instrumental (Hernandez et al., 2002), spatial (Setlow and McGaugh, 1998; Sargolini et al., 2003), and pavlovian approach learning (Dalley et al., 2005). In addition, spontaneous reactivation of neuronal firing patterns (i.e., in the absence of external stimuli) occurs in the VS during sleeping periods after a behavioral experience (Pennartz et al., 2004). In these "off-line" periods, the recurrence of neuronal activity patterns might contribute to memory consolidation by strengthening synaptic connections activated during the preceding behavior or by forming more direct connections among items stored in distributed form throughout the brain (Pavlides and Winson, 1989; Wilson and McNaughton, 1994; McNaughton, 1998; Rasch et al., 2007).

Ventral striatal reactivation, similar to hippocampal replay (Kudrimoti et al., 1999), is manifested during periods of slowwave sleep (SWS) and occurs especially in neuronal subgroups whose firing rates are modulated in close temporal association with sharp wave-ripple complexes [i.e., high-frequency oscillations in the hippocampal local field potential (LFP)] (O'Keefe and Nadel, 1978; Buzsáki, 1986). It is unknown, however, whether VS reactivation preferentially takes place during ripple episodes and whether it also occurs during rapid eye movement (REM) sleep. Interestingly, although a significant reactivation effect was reported, its occurrence and strength appeared variable across sessions (Pennartz et al., 2004).

Thus far, replay has been primarily studied in populations of hippocampal pyramidal cells exhibiting "place fields" (Wilson and McNaughton, 1994; Skaggs and McNaughton, 1996), where it may be assumed to pertain to spatial and contextual information processing. For the VS, however, the behavioral correlates of 
the reactivated information are unknown. We hypothesized that it is "reward-related" information that is reprocessed off-line in this area to endow the memory trace with a motivational component. We tested this by examining whether the VS specifically reactivates reward-related information rather than the overall ensemble spike patterns that occur during behavior in general. In addition, we examined which sleep stages exhibit this reactivation, including periods of REM.

\section{Materials and Methods \\ Experimental procedures}

Behavior. Four male Wistar rats (375-425 g; Harlan) were individually housed under a $12 \mathrm{~h}$ alternating light/dark cycle with light onset at 8:00 A.M. All experiments were conducted in the animal's inactive period. On training and recording days, intake of water was limited to a $2 \mathrm{~h}$ period after training or recording. Food was available ad libitum. Before surgery, rats were pretrained on a linear track $(185 \mathrm{~cm}$ long $\times 10 \mathrm{~cm}$ wide; $40 \mathrm{~cm}$ elevated from the floor) to shuttle back and forth for reinforcements available at both ends of the track. Over the course of training, rats were introduced to three kinds of rewards, which differed in both taste and texture [sucrose solution (10\%), vanilla desert, and chocolate mousse] and to a partial reinforcement schedule.

During recordings, rats were subjected in daily sessions to a protocol consisting of a rest period (prebehavioral rest, $20-60 \mathrm{~min}$ ) followed by a phase of reward searching behavior on a triangular track (track, 20 min) and concluded by a second period of rest (postbehavioral rest, 60-120 min). The track (equilateral sides, $90 \mathrm{~cm}$; width, $10 \mathrm{~cm}$ ) was novel to the rats at the first recording session. On the track, the rats were required to run in one direction, stopping only at reward wells positioned in the center of each arm to check whether a reward was available. To promote differential firing associated with reward sites, the three types of rewards used during pretraining were also provided to the animal in the task. Each lap, one of the three reward types could be obtained from its corresponding well. The combination of reward type and well location was fixed throughout all sessions. Rats spent the rest episodes on a towel folded in a wide flowerpot situated next to the track.

Surgery and recordings. Rats were implanted with a multielectrode microdrive containing seven individually movable tetrodes directed to the VS (1.8 $\mathrm{mm}$ anterior and $1.4 \mathrm{~mm}$ lateral to bregma) (Paxinos and Watson, 1996), whereas additional tetrodes were placed in the hippocampus. Reference electrodes were placed in the corpus callosum, and near the hippocampal fissure. A skull screw located on the caudal part of the parietal skull bone contralateral to the drive location served as ground. Spike trains from individual cells, LFPs, and the position of the rat were recorded using a 64 channel Cheetah recording system (Neuralynx). When signals exceeded a manually preset voltage threshold, waveforms were sampled at $32 \mathrm{kHz}$ for $1 \mathrm{~ms}$ (filter settings, $600-6000 \mathrm{~Hz}$ ). LFPs were continuously sampled at $1690 \mathrm{~Hz}$ and bandpass filtered between 1 and $475 \mathrm{~Hz}$. Using an array of light-emitting diodes on the headstage, a video-tracking system extracted the rat's position on the maze at $60 \mathrm{~Hz}$ with a resolution of $2.5 \mathrm{~mm} /$ pixel. The behavior of the rat was also stored on videotape. All experimental procedures were in accordance with national guidelines on animal experimentation.

\section{Data analysis}

Spike sorting. Spikes from neurons were separated from those emitted by other neurons recorded on the same tetrode by grouping spikes with similar distributions of waveform properties across the four channels of a tetrode using standard automated and manual clustering methods (i.e., Bubbleclust and MClust, respectively). BubbleClust groups spikes based on nearest-neighbor distances, clustering spikes that are close to each other, given features of the waveform such as peak amplitude or area under the curve and principal components of a spike on each tetrode channel. MClust facilitates manual selection of clusters by allowing users to limit cluster membership based on boundaries drawn on twodimensional plots of the waveform features. Clusters of spikes were attributed to a single unit on the basis of waveform characteristics and when they exhibited $<0.1 \%$ of spike intervals within a $2 \mathrm{~ms}$ refractory period in their interspike interval histograms (Fig. $1 A$ ). Units were only included in the analyses when they emitted at least 20 spikes in each behavioral/rest episode. Putative interneurons were distinguished from principal cells by means of average firing rate $(>8 \mathrm{~Hz})$ and waveform characteristics (small peak-to-valley width, valley shape) and were not included in analysis.

Identification of rest and sleep phases/ripple detection. Prebehavioral and postbehavioral rest phases comprised all periods of motionless behavior of the rats when they were situated in the flowerpot. Time frames with body movements during these episodes indicated by video tracker equipment and video tapes were extracted from the prebehavioral and postbehavioral recordings. Within these rest episodes of behavioral immobility, sleep phases were identified using LFP traces recorded near the hippocampal fissure and pyramidal layer. REM sleep was primarily defined by an elevated ratio $(>0.4)$ of spectral power density in the theta band $(6-10 \mathrm{~Hz})$ to the overall power; borders of theta oscillations were refined on secondary visual inspection. Periods of SWS were identified by the presence of large irregular activity and ripples in the hippocampal LFP (Vanderwolf, 1969; O’Keefe and Nadel, 1978; Buzsáki, 1986; Kudrimoti et al., 1999; Pennartz et al. 2004). After filtering a LFP trace from the pyramidal cell layer between 100 and $300 \mathrm{~Hz}$, a ripple was detected each time the squared LFP trace exceeded a preset threshold (3.5 SD) for at least $25 \mathrm{~ms}$. Adjacent ripples were merged when the ripple interval was $<100 \mathrm{~ms}$. The presence of large irregular activity in the LFP was verified on off-line visual inspection. Short periods of quiet wakefulness may have been included in SWS episodes because the LFP patterns of both states share principal features. For this reason, this state is further referred to as quiet wakefulness (QW)-SWS. Rest periods lasting shorter than $20 \mathrm{~s}$ were excluded from classification and additional analysis. Thus, rest periods contained QW-SWS and REM sleep as main components, whereas segments of unclassified rest period constituted a very minor part of rest. Periods of active wakefulness within the rest episodes were not analyzed as a separate state of the sleep-wake cycle because the periods were generally short and did not contain sufficient spike counts.

Reward-related firing patterns. To identify reward-related units, perievent time histograms were constructed for the rewarded and nonrewarded condition for each reward site. The histograms were synchronized on reward site arrivals, which were signaled by the crossings of off-line installed "virtual photobeams," positioned right before the point at which the rat reached each reward well. Reward-related responses were assessed within a period of $1 \mathrm{~s}$ before and $1 \mathrm{~s}$ after arrival at a reward site. Spike counts were binned in $250 \mathrm{~ms}$ intervals. The eight bins comprising the reward period were each compared with three bins taken from the corner passage opposite to the well under scrutiny within the same lap (Wilcoxon's matched-pairs signed rank test, $p<0.01$ ). A bin of the reward period was only considered significantly different when the rank test (which includes as entries a list of all spike count values from the test bin paired with those from the control bin per reward period) indicated significance from each of the three control bins. We verified that the firing in the control period of three bins was not marked by specific deviations from the firing in all intermediate segments between corners and reward sites using perievent time histograms and plots of the spatial distribution of firing rates. Responses were qualified as significant when one or more bins in the reward period were significantly different from each of the three reference bins. This control period was preferred over, for example, the average firing rate per lap because many neurons were virtually silent during track running except for their brief, phasic response at one or more reward sites. Thus, the average firing rate of these cells strongly depends on the response intensity itself, which would enhance the bias toward false-negative responses (i.e., erroneously identified as nonresponsive) if it were used as control value. However, results were comparable when other control measures were used such as the baseline firing rate or the average firing rate per lap. Differences between responses at the three reward sites were statistically evaluated with a Kruskal-Wallis test $(p<0.05)$ followed by a Mann-Whitney $U(M W U)$ test $(p<0.05)$, whereas rewarded versus nonrewarded conditions were compared using MWU $(p<0.05)$.

Quantification of reactivation. The assessment of covariation in firing rates and the quantification of reactivation with the explained variance method was previously described (Kleinbaum et al., 1998; Kudrimoti et 
A
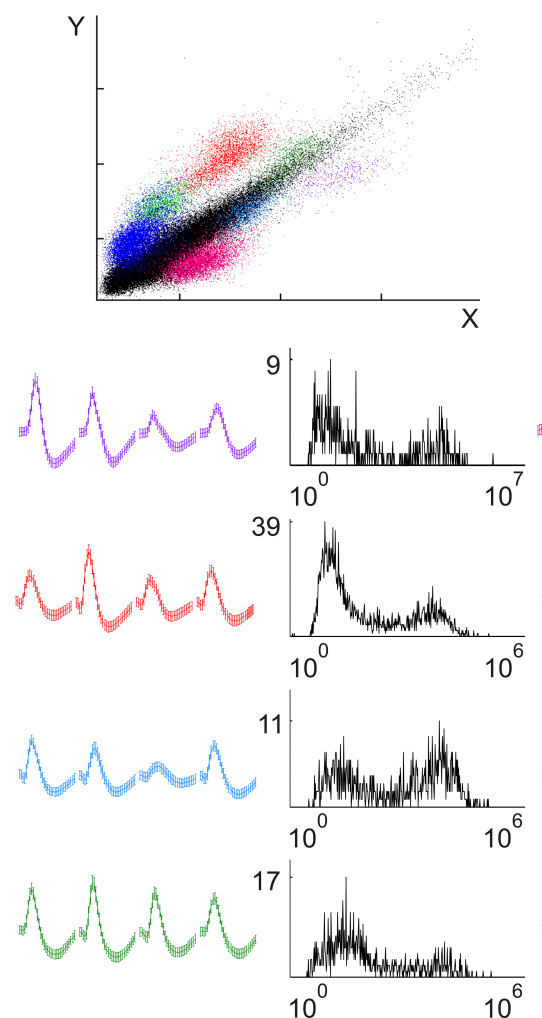
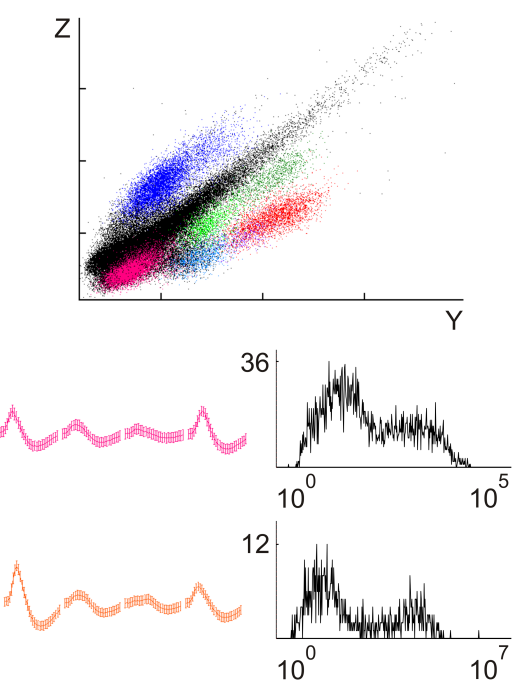

12

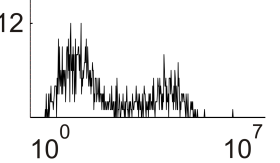

13.
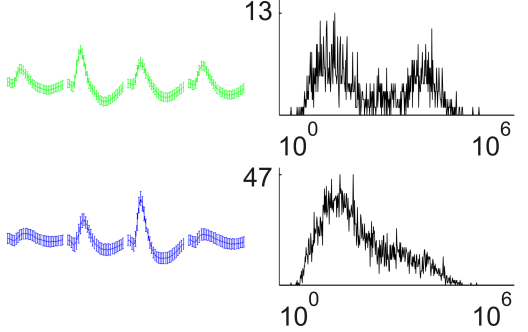

B
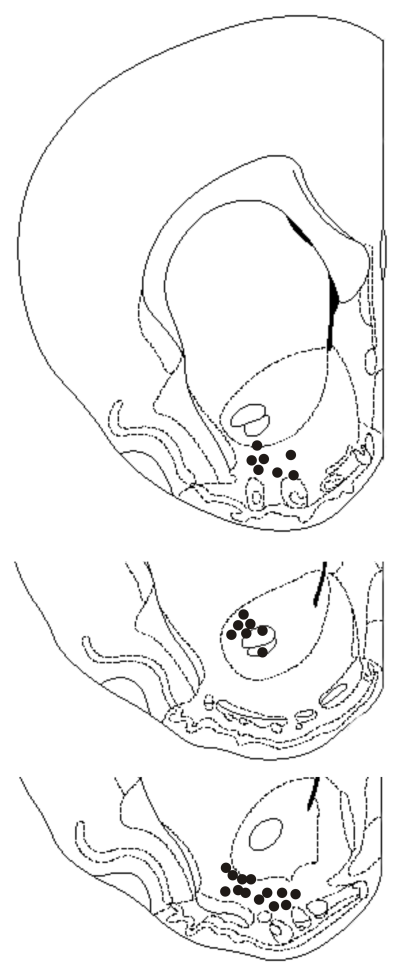

Figure 1. $\boldsymbol{A}$, Example of a tetrode recording: projection plots of cluster isolation, waveforms, and interspike interval (ISI) histograms. Spikes belonging to separate clusters were identified according to multiple waveform features including area under the curve (plotted in this figure), peak amplitude, and principal components. $X, Y$, and $Z$ correspond to three of four leads of the tetrode. The color of the waveforms shown in the first and third column (bottom) corresponds to the colors of the individual clusters in the projection plots. Top, The black diagonal band corresponds to unclassified events, including noise. Bottom, In the second and fourth column, ISI histograms are shown for each unit represented to the left, with ISI counts on the ordinate and interval duration on the abscissa. $\boldsymbol{B}$, Schematic representation of the endpoints of the tetrode tips. The tetrode endings were in the ventral striatum approximately between 2.2 and 1.2 anterior to bregma and between 1.6 and 3.0 laterally compared with an atlas of the rat brain (Paxinos and Watson, 1996).

al., 1999; Pennartz et al., 2004; Tatsuno et al., 2006). Briefly, spike trains of simultaneously recorded neurons were binned in intervals of $50 \mathrm{~ms}$ to obtain sequences of spike counts for each episode. Temporal correlations of the firing patterns of neuron pairs were determined by computing Pearson's correlation coefficients for each episode separately. All coefficients of a particular rest/active episode were assembled into a single matrix and the similarity between the three matrices was determined by computing a correlation coefficient for each of three possible combinations of two rest/active episodes. These matrix-based correlation coefficients were used to determine the degree to which the variance in the correlation pattern in postbehavioral rest can be explained by the pattern established during the behavioral experience while factoring out any correlations present before the behavioral experience. This quantity is expressed in the explained variance (EV) measure as follows:

$$
\mathrm{EV}=r_{\text {Track, R2/R1 }}^{2}=\left(\frac{r_{\text {Track, R2 }}-r_{\text {Track, } \mathrm{R} 1} r_{\mathrm{R} 2, \mathrm{R} 1}}{\sqrt{\left(1-r_{\text {Track, } \mathrm{R} 1}\right)\left(1-r_{\mathrm{R} 2, \mathrm{R} 1}^{2}\right)}}\right)^{2},
$$

where $\mathrm{R} 1$ is the prebehavioral rest phase and $\mathrm{R} 2$ is the postbehavioral rest phase. For example $r_{\text {Track,R2 }}$ equals the matrix-based correlation between the track running and postbehavioral rest pattern. EV equals the square of the partial correlation coefficient and is bounded between 0 and 1 . As a within-subject and session control measure, the reverse explained variance (REV) can be computed by swapping R1 and R2 in the previous equation, thereby switching the temporal order of episodes. EV and REV values were computed for all recorded sessions that contained at least five well isolated active neurons and for time blocks of 20 min composed of quiet rest and sleep (i.e., periods of active behavior were excluded). Therefore, correlated firing between cells caused by behaviors irrelevant to the task, such as grooming, cannot influence reactivation measures.
Sessions that showed reactivation $(\mathrm{EV}>\mathrm{REV})$ in the first 20 min rest block after track running were used to assess decay and the contribution of individual cell pairs to the session EV. Two control procedures were performed to check whether the observed reactivation was time- and cell-specific (Louie and Wilson, 2001): (1) entire spike train vectors from the behavioral episode were temporally shifted relative to the original time stamps of the same cell. The shift was circular, so that data removed from the pattern at one end were reinserted at the opposite end; the temporal distance ranged between $2 \mathrm{~s}$ forward and backward and varied randomly among cells. (2) Entire spike train vectors from the behavioral episode were randomly reassigned between cells. In both cases, the temporal order of spike activity within each train was preserved. Differences between the EV and REV session values were statistically assessed with Wilcoxon's matched-pairs signed rank test.

For reactivation analysis of subgroups of neurons, Pearson's correlation coefficients obtained from all rats across all sessions were pooled, and subsequently EV and REV values were determined. To obtain estimates of the mean and variance of the $\mathrm{EV}$ and $\mathrm{REV}$ values, a bootstrapping procedure was applied in which randomly drawn samples were generated $(n=10,000)$ from the observed set of correlation coefficients (Sokal and Rohlf, 1995) (cf. Hoffman and McNaughton, 2002). The resampling procedure was done with replacement so that each sample may contain repetitions of some triplets and omissions of others. Random samples were of the same size as the original and triplets of correlation coefficients obtained for the three task episodes (i.e., from the prebehavioral rest, running period, and postbehavioral rest) of a single recording were kept together during the resampling. Reactivation measures were computed for each sample resulting in distributions of estimated EV and REV values for each subset. Differences between the means of the distributions of subsets were statistically evaluated with the MWU test. 
Temporal order of firing. We used temporal bias (Skaggs and McNaughton, 1996) and sliding template (Louie and Wilson, 2001; Tatsuno et al., 2006) analyses to assess whether the temporal order of firing within striatal cell pairs was preserved from track running to the postbehavioral rest episode as was previously described for the hippocampus. However, probably because of the limited number of strongly reactivating cell pairs in each session and the generally low firing rates of the neurons, these analyses did not yield additional results shedding light on this question.

\section{Histology}

The final position of the tetrodes was marked by passing a $25 \mu \mathrm{A}$ current lasting $10 \mathrm{~s}$ through one lead of each channel to produce a small lesion. The next day, animals were transcardially perfused with a $0.9 \% \mathrm{NaCl}$ solution followed by $4 \%$ paraformaldehyde in PBS $(0.1 \mathrm{M})$, $\mathrm{pH} 7.4$, before the brains were removed. Coronal brain sections $(40 \mu \mathrm{m})$ were cut on a Vibratome and Nissl-stained for verification of tetrode tracks and end points. All of the tetrodes endings were in the ventral striatum approximately between 2.2 and 1.2 anterior to bregma and between 1.6 and 3.0 laterally compared with an atlas of the rat brain (Paxinos and Watson, 1996). To estimate the number of recordings originating from the core and shell subdivisions of the ventral striatum, we first assessed the endpoints of the individual tetrodes in the histological sections and converted them to coordinates according to the atlas (Fig. $1 B$ ). We then calculated the approximate depths of the tetrodes in each session by subtracting the estimated travel distance from the tetrode endpoint. Thirty sessions yielded recordings from 203 locations, of which 109 (54\%) were likely in the core and 94 (46\%) in the shell region. Note that ensembles from most sessions were likely to contain both core and shell recordings. Six recording sessions were identified as containing coreonly recordings, and reactivation was present in these sessions.

\section{Results}

Activity of multiple single units in the VS was monitored in four rats during daily episodes of reward-searching behavior on a triangle track flanked by two rest periods. On the track, rats typically ran from well to well and stopped each time checking for reward availability. As the rats became proficient at acquiring rewards on the track, the number of laps ran in 20 min increased over sessions $(15.3 \pm 4.1$ in the first to $62.3 \pm 6.2$ in the 10 th; linear regression $R^{2}=0.53 ; p<0.0001$ ) (supplemental Fig. $1 \mathrm{~A}$, available at www.jneurosci.org as supplemental material). The travel time between two wells was significantly longer when a reward was consumed than when an empty well was visited (16.54 \pm 0.38 and $7.65 \pm 0.25 \mathrm{~s}$; MWU, $p<0.0001)$. The interwell intervals did not become significantly shorter when several empty wells were encountered consecutively.

\section{Behavioral correlates of ventral striatal firing patterns}

A total of 398 well isolated, stable, and sufficiently active single units was recorded over 30 sessions ( $13.3 \pm 0.9$ per session). Of these neurons, 79 (19.8\%) showed significant firing rate changes correlated to reward site visits, whereas $7(1.8 \%)$ showed changes to other task components such as locomotion. Responses timelocked to reward site visits were generated both by putative fastspiking interneurons $(n=13)$, which were not included in the analyses below, and other neurons (probably mostly mediumsized spiny neurons; $n=66$ recorded from 25 sessions; 5 sessions recorded from three rats did not contain any reward-related correlates). Neurons showing reward-related correlates exhibited various firing profiles (Fig. 2, Table 1). They responded predominantly by increasing their firing rates $(61 ; 92.4 \%)$, whereas occasionally firing rate decrements were observed (5; 7.6\%). Individual responses peaked before the rat's arrival at a well $(20 ; 30.3 \%)$, during the postarrival phase $(20 ; 30.3 \%)$, or consisted of elevations during both phases $(26 ; 39.3 \%)$ (supplemental Fig. 2, available at www.jneurosci.org as supplemental material). More neu- rons changed firing rates when a reward was present $(39 ; 59.1 \%)$ than when it was absent $(9 ; 13.6 \%)$. Five neurons $(7.6 \%)$ showed firing rate changes in both conditions but with significantly different response magnitudes, whereas the remaining units showed no difference between presence and absence of reward. About one-half of the neurons discriminated between reward sites, by either selectively responding to a single site $(21 ; 31.8 \%)$, two sites $(9 ; 13.6 \%)$, or to all three sites $(6 ; 9.1 \%)$. In the latter two cases, responses to individual reward sites could differ in magnitude ( 9 of 15 units). Interestingly, a subset of neurons (26; 39.3\%) discriminated between the reward versus no-reward condition as well as between different reward sites. A last subset of cells (8; $12.1 \%$ ) was less selective in their firing profiles in that their firing rate changes reached significance only when all reward conditions (absence/presence and sites) were lumped together.

In line with previous studies on rat VS (Roitman et al., 2005; Tran et al., 2005), we will apply the term reward-related to all units showing significant responses time-locked to reward site visits. Three units responded to one reward site only and, in addition, did not fire differentially for reward presence versus absence. Although these cells were included in our analysis as reward related, it cannot be excluded that their firing is purely spatially modulated (Lavoie and Mizumori, 1994; Shibata et al., 2001). However, exclusion of these units from analysis yielded similar results. When the firing patterns of reward-related and other units were viewed together during track running, sequences of consecutively firing neurons were observed, which, however, showed great variability because of time-varying allocation of reward to the three sites (Fig. $3 A$ ). Figure $3 B$ shows the same ensemble firing in parallel with hippocampal local field potential during subsequent QW-SWS.

\section{Reactivation in the ventral striatum}

The ventral striatum showed reactivation (EV, $17.6 \pm 4.1 \%$; REV, $5.2 \pm 1.3 \% ; p<0.001 ; n=30$ ) (Fig. 4), which is in line with previous results (Pennartz et al., 2004). The EV exceeded the REV in a majority of sessions (23 of 30 ). Including putative fastspiking interneurons in the analysis yielded similar reactivation values (EV, $15.9 \pm 3.2 \%$; REV , $4.4 \pm 1.1 \%$; $p<0.01$ ). When the correlations between cells recorded on the same tetrode were removed from the analysis, we still observed a significant reactivation $(\mathrm{EV}, 11.0 \pm 3.6 \%$; REV, $3.7 \pm 1.0 \%$; $p<0.05)$. To examine whether the observed reactivation was attributable to celland time-specific firing correlations, EV and REV values were recalculated after the spike trains of the behavioral episode had been randomized in two different ways (Louie and Wilson, 2001). Both (1) randomly shifting these spike trains in time and (2) reassigning these spike trains to different cells strongly decreased EV values and removed the significant difference between EV and REV (1: EV, $3.3 \pm 0.7 \%$; REV, $2.8 \pm 0.2 \%$; $:$ EV, $2.1 \pm$ 0.9\%; REV, $2.1 \pm 0.9 \%$; NS) (Fig. 4A). Reactivation measures in these control procedures were significantly lower than the original values $(p<0.002)$. Furthermore, the strength of reactivation was significantly correlated with the progression through the sessions [linear regression on the difference $(\mathrm{EV}-\mathrm{REV}), R^{2}=0.24$; $p<0.01]$ and with the number of laps ran on the track $\left(R^{2}=\right.$ $0.17 ; p<0.05$ ) (supplemental Fig. $1 B$, available at www. jneurosci.org as supplemental material). Because the detectability of reactivation may positively correlate with the behavioral regularity and repetitiveness of task performance, this positive correlation per se does not confirm or contradict a role for reactivation in learning and memory consolidation (Jackson et al., 2006). 
A

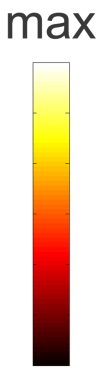

$\min$

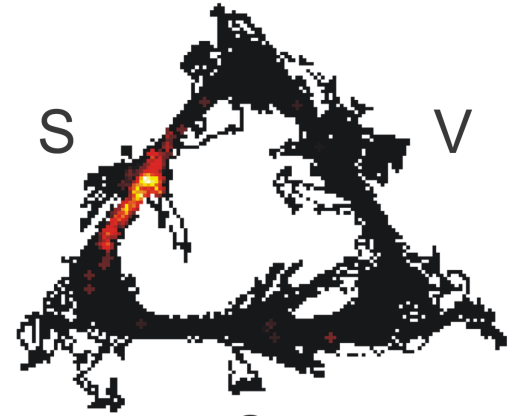

C


B
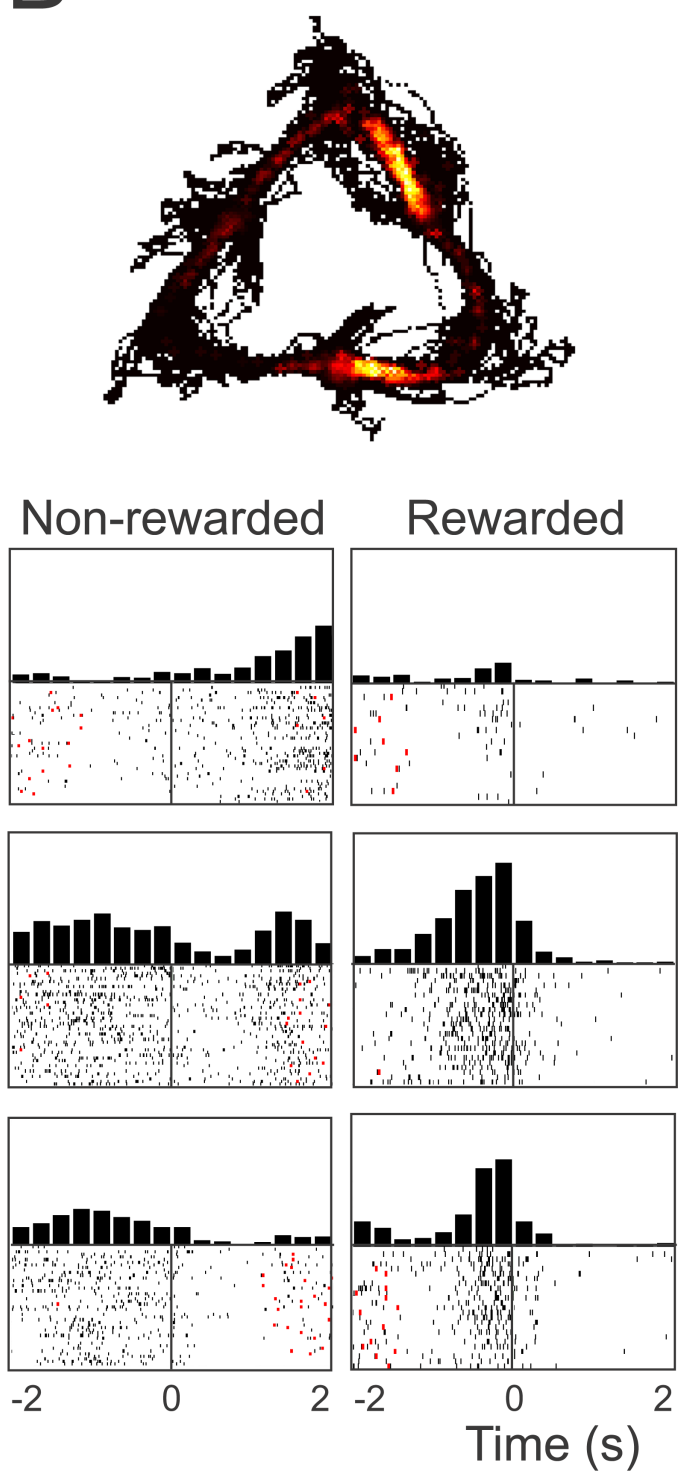

Figure 2. Ventral striatal units showing firing patterns associated with one or more reward sites. Rats ran along a triangular track to obtain three types of reward (S, sucrose; $V$, vanilla; $C$, chocolate). Top panels, Spatial distribution of firing rates of two individual neurons. $\boldsymbol{A}, \boldsymbol{B}$, Local firing rates ranged from 0 to maxima of $19 \mathrm{~Hz}(\boldsymbol{A})$ and $18 \mathrm{~Hz}(\boldsymbol{B})$. Bottom panels, Perievent time histograms for both cells synchronized on reward site arrivals. Rows represent different reward types, whereas columns differentiate between the presence and absence of reward. Firing rate is in bins of $250 \mathrm{~ms}$. The red ticks indicate arrivals at other reward sites. $\boldsymbol{A}$, Neuron showing an increased firing rate shortly before and after arriving at the sucrose reward site, mainly when a reward was obtained. $\boldsymbol{B}$, Neuron increased its firing rate before arrival at two sites differentially for the rewarded versus nonrewarded condition. The response at the vanilla site was stronger than at the chocolate site. The rat may have detected the availability of reward before arriving at the well by visual or olfactory cues, although reward cups were filled outside the rat's field of view.

The dynamics of reactivation in the course of time after track running was studied by comparing two 20 min blocks of concatenated quiet rest/sleep (block 1: EV, $22.2 \pm 4.3 \%$; REV, $4.0 \pm$ $1.3 \%$; $p<0.0001$; block 2: EV, $16.6 \pm 4.2 \%$; REV, $7.7 \pm 2.5 \%$; $p<0.002)$. We did not find a significant decline over the two blocks (NS). Because periods of awake behavior within postexperiential rest were excluded from this analysis, the amount of rest time in which rats slept for a total of $40 \mathrm{~min}$ was $53.7 \pm 1.7 \mathrm{~min}$. Similar results were obtained when reactivation was computed over continuous $20 \mathrm{~min}$ time blocks, taking only the quiet rest/ sleep periods within the blocks into account.

Ventral striatal reactivation occurs in SWS, but whether it emerges during REM sleep has remained unclear (Pennartz et al., 2004). To address this issue, REM sleep and QW-SWS episodes were delineated in 16 sessions containing at least 4 min of REM sleep (prebehavioral rest, $8.0 \pm 0.7$; postbehavioral rest, $10.2 \pm$ $0.9 \mathrm{~min}$ ) and QW-SWS (prebehavioral rest, $20.4 \pm 2.0$; postbehavioral rest, $37.4 \pm 2.9 \mathrm{~min}$ ) per rest episode (Fig. $4 \mathrm{~B}$ ). Reactivation during QW-SWS was comparable in strength to the replay computed across whole rest periods in the same sessions (EV, $16.3 \pm 4.7 \%$; REV , $5.1 \pm 1.6 \% ; p<0.02)$. Specifically, reactivation appeared particularly strong during short time windows (200 ms) after ripple onset (EV, $22.3 \pm 5.3 \%$; REV, $2.8 \pm 3.8 \%$; $p<0.001$ ), whereas for windows of identical length taken from the intervals between ripples the EV was not significantly different from the REV (EV, $13.0 \pm 4.7 \%$; REV, $8.8 \pm 3.1 \%$ ). Reactivation for spikes within the ripple windows was significantly different from spikes in interval windows $(p<0.01$ for both EV and the difference EV - REV). We noted that the duration of ripples in postbehavioral QW-SWS was slightly but significantly in- 
Table 1. Numbers of reward-related responses

\begin{tabular}{|c|c|c|c|}
\hline & No. of cells & $\begin{array}{l}\text { Total recorded } \\
\text { cells }(\%)\end{array}$ & $\begin{array}{l}\text { Reward-related } \\
\text { responses (\%) }\end{array}$ \\
\hline Total no. of recorded cells & 385 & 100 & \\
\hline No. of reward-related units & 66 & 17.1 & 100 \\
\hline \multicolumn{4}{|l|}{ Firing rate response } \\
\hline Increase & 61 & & 92.4 \\
\hline Decrease & 5 & & 7.6 \\
\hline \multicolumn{4}{|l|}{ Response timing relative to reward site arrival } \\
\hline Prior to arrival & 20 & & 30.3 \\
\hline After arrival & 20 & & 30.3 \\
\hline During both phases & 26 & & 39.3 \\
\hline \multicolumn{4}{|l|}{ Reward availability } \\
\hline Reward present & 39 & & 59.1 \\
\hline Reward absent & 9 & & 13.6 \\
\hline Differential responses between conditions & 5 & & 7.6 \\
\hline \multicolumn{4}{|l|}{ Reward sites } \\
\hline 1 site & 21 & & 31.8 \\
\hline 2 sites & 9 & & 13.6 \\
\hline 3 sites & 6 & & 9.1 \\
\hline Differential responses between sites & $9 / 15$ & & 60.0 \\
\hline \multicolumn{4}{|l|}{ Reward availability and reward sites } \\
\hline Differential firing to presence and sites & 26 & & 39.3 \\
\hline Firing pattern not specific for any condition & 8 & & 12.1 \\
\hline \multicolumn{4}{|c|}{$\begin{array}{l}\text { Shown is an overview of the number of significant reward-related correlates. A total of } 66 \text { reward-related correlates were identified from } 385 \text { units (Wilcox- } \\
\text { on's matched-pairs signed rank test, } p<0.01 \text { ). Subsets of reward-related correlates, classified under "Reward availability" and "Reward sites," responded } \\
\text { differentially to the presence/absence of reward or to the reward sites, respectively, whereas the remaining neurons were less selective in their firing profiles. } \\
\text { The term "differential responses" between reward presence and reward absence indicates that, in both conditions, a significant response was observed but } \\
\text { the amplitude of the responses was significantly different (Kruskall-Wallis, } p<0.05 \text {; Mann-Whitney } U \text { test, } p<0.05 \text { ). The same specification holds for } \\
\text { differential responses between reward sites. Some neurons discriminated between reward presence/absence and between reward sites. These are listed } \\
\text { under "Differential firing to presence and sites." }\end{array}$} \\
\hline
\end{tabular}

quires at minimum 2 cells per session, and therefore fewer RRUs $(n=57)$ could be used than recorded throughout all 25 sessions. The RRU group showed an extremely strong reactivation (EV, 50.8\%; REV, $0.3 \% ; n=57$ cells), whereas the NRU group $(n=166)$ yielded a much smaller EV value (i.e., 14.6\%) and REV $(2.3 \%)$. To assess significance, we applied a bootstrapping procedure with resampling of pooled correlation values. The distributions showed a significantly higher EV than REV in both subsets $(p<0.001)$, demonstrating reactivation in both ensembles (Fig. 5A). Moreover, the mean reactivation of the RRU subset was significantly higher than the NRU group as assessed from the distributions of the difference EV minus REV (EV - REV, $p<$ $0.001)$.

The $(\mathrm{EV}-\mathrm{REV})$ distribution of the RRU group, however, was broad and bimodal (Fig. 5A). This suggests that cell pairs are unevenly contributing to the observed reactivation. To estimate the relative contribution of each cell pair to the session reactivation, a pair was excluded from the population after which EV and REV values were recomputed. The difference between the session EV minus the EV after pair exclusion represents the estimated contribution of that pair to the ses-

creased compared with prebehavioral QW-SWS $(99.0 \pm 0.4$ and $95.3 \pm 0.3 \mathrm{~ms}$, respectively; $p<0.02$ ). The emission rate of ripples was similar in both prebehavioral and postbehavioral QWSWS (prebehavioral SWS, $0.65 \pm 0.01 \mathrm{~Hz}$; postbehavioral SWS, $0.66 \pm 0.01 \mathrm{~Hz}$; NS). Significant reactivation was not detected in REM sleep (EV, $7.5 \pm 2.5 \%$; REV, $3.8 \pm 0.8 \%$; NS). When bin sizes $>50 \mathrm{~ms}$ were used to capture one or more theta cycles, similar results were obtained. To control for possibly confounding effects caused by undersampling of REM sleep or the timing of REM sleep throughout the resting period, reactivation was computed over QW-SWS episodes that were equal in length to REM episodes and followed these in time. These episodes also showed significant reactivation (EV, $13.2 \pm 3.5 \%$; REV, $4.4 \pm$ $1.1 \% ; p<0.05)$, similar to the amounts found for total QW-SWS time. The mean firing rate across all cells was significantly higher in REM sleep than in QW-SWS $(0.34 \pm 0.02$ and $0.23 \pm 0.01 \mathrm{~Hz}$, respectively). Correspondingly, the lack of REM sleep reactivation could not be ascribed to an undersampling in terms of total spike numbers (REM prebehavioral rest, $2165 \pm 269$; postbehavioral rest, $2645 \pm 396$; QW-SWS prebehavioral rest, $1319 \pm 192$; postbehavioral rest, $1836 \pm 231$ ).

\section{Reactivation of motivationally relevant information}

We next addressed the question whether reward-related information was specifically reactivated in the VS. Reactivation was assessed for the subset of reward-related units (RRUs) and the subset of units recorded in the same sessions without such correlates [nonrelated units (NRUs)]. The number of RRUs per session was generally low ( $2.6 \pm 0.3 ; n=25$ sessions). Therefore, all cell pair-based Pearson's correlations per episode were pooled across sessions and animals for each subset. This procedure re- sion EV. Only 5 of 70 cell pairs $(7.1 \%)$ contributed $>10 \%$ to their respective $\mathrm{EV}$, but there were no pairs that negatively contributed to the EV by more than $-6 \%$ (binomial test, $p<0.05$ ) (Fig. $5 B, C)$. These five highly contributing cell pairs were all recorded from different sessions across four rats. All but one of these 10 cells showed increased firing rates before the rat's arrival at reward wells. Generally, the rate maps and perievent time histograms of two cells forming a highly contributing pair were similar, and their cross-correlograms showed a prominent peak around zero, indicating a high degree of synchronous firing during track running and during the subsequent rest period (Fig. $5 C)$. When all of the highly contributing pairs were excluded from the RRU group, the pooled EV value dropped to $0.63 \%$ ( $\mathrm{REV}, 7.5 \%$ ), indicating that reactivation in the RRU group strongly depended on a small fraction of the RRU population (Fig. 5D). The incidences of high contributors $(\geq 10 \% \mathrm{EV})$ in the NRU pairs and the pairs consisting of one RRU and one NRU (mixed pairs) appeared lower than in the RRU group [NRU, 7 of $1379(0.005 \%)$; mixed, 3 of $545(0.006 \%)]$ and were not significantly different from the incidences of pairs that made a large negative contribution to the EV (i.e., less than or equal to - 10\%) in both groups. Reactivation was still present in the NRU group when the high contributors were excluded from the population (EV, 8.8\%; REV, 2.3\%; $p<0.001$ ); however, this residual replay was weaker than in the total NRU population.

In principle, differences in the number of cell pairs, average firing rates, or correlation strength between spike trains during the behavioral experience between the subgroups could account for the observed difference in reactivation between the RRU and the NRU groups (Fig. 5E). To examine these possibilities, the RRU group (mean firing rate, $1.3 \pm 0.2 \mathrm{~Hz}$; mean correlation 
strength, $0.033 \pm 0.007$ ) was tested against three subsets of the NRU group that were matched for number of cell pairs and (1) were taken from the same sessions (session-matched NRU), (2) showed similar mean firing rate during track running $(1.2 \pm 0.1 \mathrm{~Hz}$, rate-matched NRU), or (3) showed similar mean and distribution of Pearson's correlation coefficients $(0.034 \pm$ 0.004 , correlation-matched NRU). The NRUs comprising the various control groups showed similar waveform characteristics and interspike interval (ISI) distributions as the RRUs. In line with the overall NRU group, reactivation was apparent in all three groups, most prominently in the correlation-matched NRU (EV, $33.6 \%$; REV , 1.3\%) and the rate-matched NRU (EV, 31.6\%; REV, 5.5\%) but also in the session-matched NRU (EV, 22.0\%; REV, $0.4 \%$; all groups; $p<0.001)$. Reactivation in all three of these NRU subgroups, however, was not nearly as strong as in the RRU group $(p<0.001)$.

The results presented thus far leave open the possibility that the reactivation observed for RRUs is not attributable to neural activity temporally linked to reward site visits but to intervals between them. Thus, we examined reactivation measures based on the narrow time windows during track running that were associated with reward site visits $(-0.5$ to +0.5 s relative to site arrival), and found a significantly higher EV than REV when pairwise spike train correlations within these windows were compared with prebehavioral and postbehavioral rest episodes (EV, 36.9\%; REV, $0.38 \%$; $p<0.001$ ) (Fig. $5 F$ ). Reactivation occurred also in the intervals between these windows but was significantly less when either the total duration (EV, $14.6 \%$; REV , $0.2 \%$; $p<0.001$ ) or the spike counts (EV, $17.8 \%$; REV, $1.2 \%$; $p<0.001$ ) were comparable with the reward-related window (reward site visits vs intervals, $p<$ 0.001 ). Time windows ranging from 200 to $750 \mathrm{~ms}$ before and after reward site visits and corresponding intervals yielded similar results (Fig. $5 F$ ). The session-matched NRUs also reactivated both during the reward site visits (EV, 19.4\%; REV, 6.6\%; $p<0.001)$ and intervals (EV, $10.6 \%$; REV, $0.9 \% ; p<0.001)$. Although reactivation was stronger during the reward site visits $(p<0.001)$, the difference was less prominent than for the RRUs. None of the other NRU groups showed the same pattern. In the overall NRU group, the rate-matched NRU group and the correlation-matched group reactivation was absent during both the reward site visits (overall NRU: EV, 1.2\%; REV, $0.0 \%$; ratematched NRU: EV, $1.0 \%$; REV , $0.8 \%$; correlation-matched NRU: $\mathrm{EV}, 2.3 \%$; REV , 0.8\%) and the intervals (overall NRU: EV, 3.1\%; REV, $0.0 \%$ ) (Fig. $5 F$ ) (rate-matched NRU: EV, 0.2\%; REV, 3.0\%;

B: QW-SWS

\section{A: Track running}
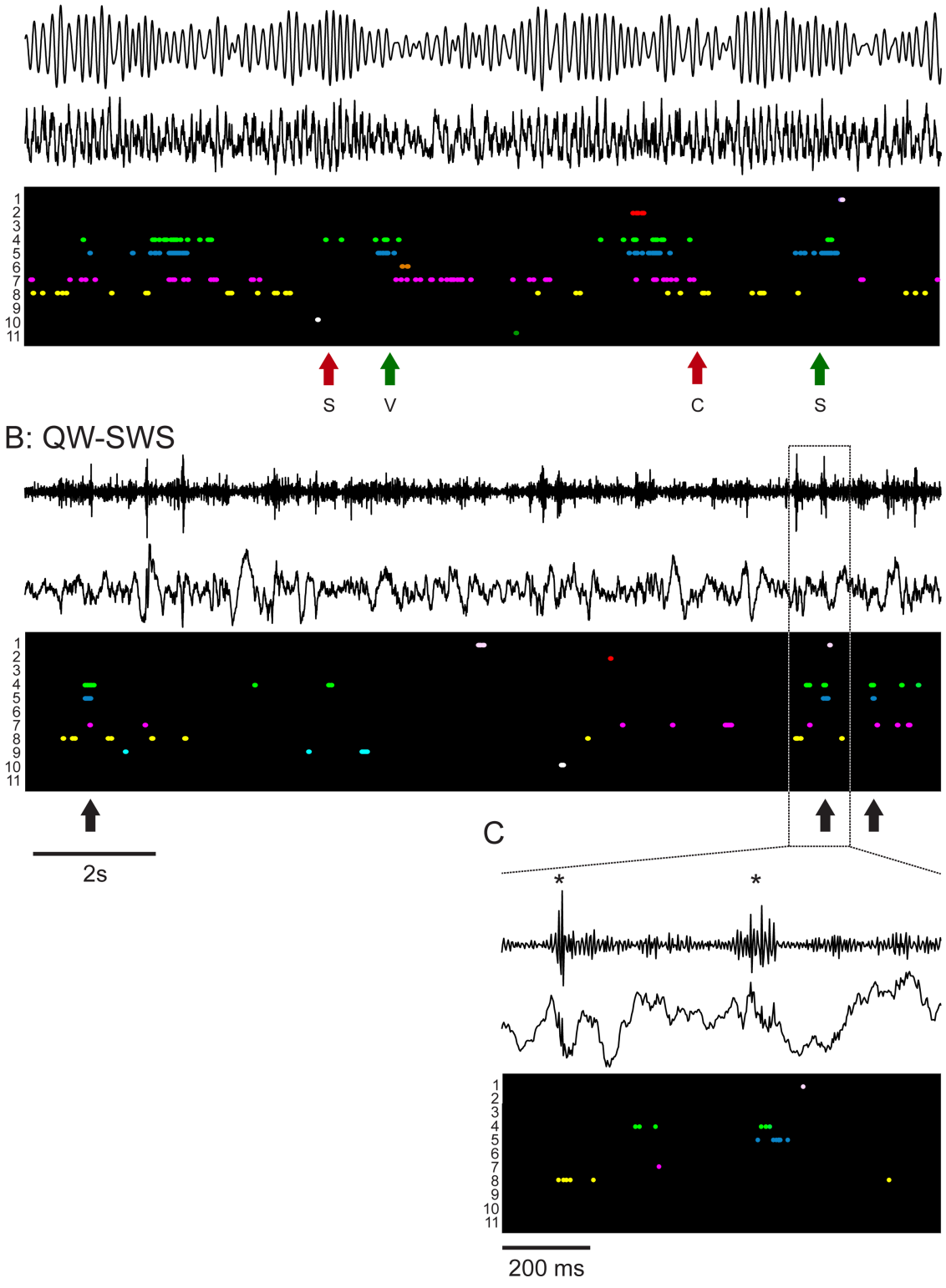

Figure 3. $\boldsymbol{A}-\boldsymbol{C}$, Examples of the firing patterns of a ventral striatal ensemble, plotted in parallel with the local field potential recorded near the hippocampal fissure $(\boldsymbol{A})$ and the pyramidal cell layer $(\boldsymbol{B}, \boldsymbol{C})$. $\boldsymbol{A}$, As the rat is running on the track, theta oscillations were observed in the hippocampal field potentials. The bottom and top traces represented raw and filtered $(6-10 \mathrm{~Hz})$ field potentials, respectively. In a period of $15 \mathrm{~s}$, the rat crossed four reward sites (s, sucrose; v, vanilla dessert; $c$, chocolate mousse), two of which were baited (indicated with green arrows) and two of which were not (red arrows). Each row in the plot below the field potential traces represents a single unit; its spikes are marked by specifically colored dots. Note the high variability in the firing patterns around each reward site arrival but also the positively correlated firing of the "green" (4) and "blue" (5) units. B, The hippocampal local field potential during QW-SWS is dominated by large irregular activity interleaved with sharp wave-ripple complexes. The bottom and top traces represent raw and filtered $(100-250 \mathrm{~Hz})$ field potentials. Note the concurrent firing of the green (4) and blue (5) units (two high contributors) indicated by black arrows. C, Enlargement of a segment of the LFP traces and spike patterns shown in $\boldsymbol{B}$. The firing of units 4 and 5 as well as spikes of unit 8 are aligned to identified ripples in the hippocampal LFP, which are marked with asterisks.

correlation-matched NRU: EV, 0.1\%; REV, 0.1\%). An increase of the time period flanking reward site arrivals and intervals of at least several seconds was required to obtain a reactivation strength comparable with that found for the whole track running period.

Next, we tested whether the strong reactivation in the RRU group specifically occurred during a particular sleep phase. For QW-SWS, the EV (77.1\%) greatly exceeded the REV (7.4\%; 

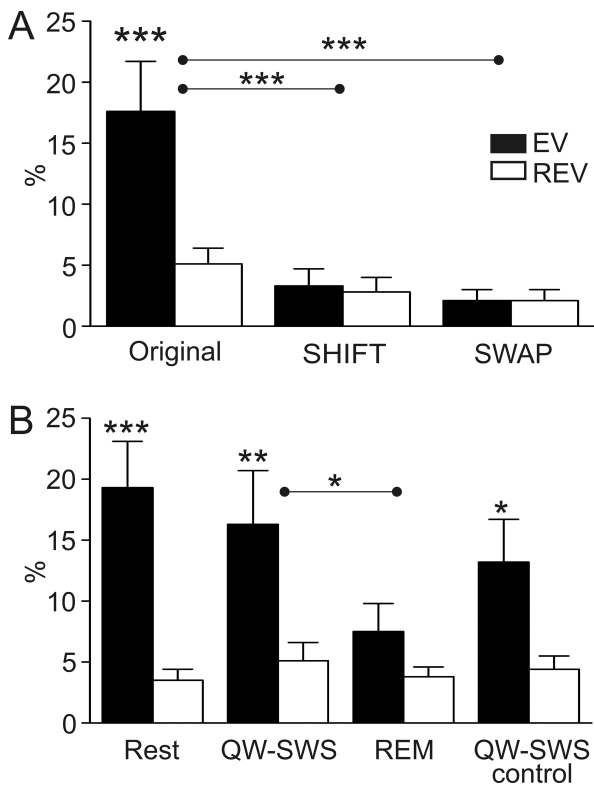

Figure 4. Reactivation in the ventral striatum: general characteristics and sleep phases. $\boldsymbol{A}$, The EV exceeded the REV when compared across sessions (original: ${ }^{* *} p<0.001 ; n=30$ ). Reactivation disappeared when the temporal alignment between spike trains was disrupted

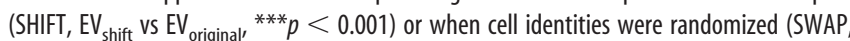
$\mathrm{EV}_{\text {swap }}$ vs $\left.\mathrm{EV}_{\text {original }}{ }^{* * *} \mathrm{p}<0.001\right)$. B, Reactivation observed during QW-SWS sleep $\left({ }^{* *} p<\right.$ 0.02 ) was not different from the reactivation found for the entire rest episodes, which included all episodes of motionless behavior (rest, $n=16$ sessions). In contrast, REM sleep periods did not show reactivation and EV values were significantly lower than for QW-SWS $\left({ }^{*} p<0.05\right)$. This lack of reactivation cannot be ascribed to the relatively late occurrence of REM sleep periods after sleep onset because post-REM QW-SWS periods showed significant reactivation (QW-SWS control, $\left.{ }^{*} p<0.05\right)$. The strength of the observed reactivation during these intervals was not significantly different from the complete QW-SWS periods. Error bars indicate SEM.

MWU, $p<0.001)$. In contrast, for REM sleep, low EV and REV values were found, 1.3 and $0.8 \%$, respectively (NS). The computation of RRU reactivation over QW-SWS episodes that were equal in length to REM episodes and followed these in time yielded an EV and REV of 55.4 and $0.1 \%$ (MWU, $p<0.001$, relative to REM sleep). Similar to the results for the complete population per session, this difference between QW-SWS and REM sleep for RRUs could not be explained by spike count (REM R1, 759.6 \pm 138.9; REM R2, $991.2 \pm$ 204.4; QW-SWS R1, 407.1 \pm 107.3; QW-SWS R2, $710.1 \pm 155.5$ ), duration, or temporal order effects.

In addition to the preferential reactivation of reward-related firing patterns, there may be a concomitant experiencedependent change in their temporal relationships with ripples. Therefore, we considered a measure of the proportion of spikes occurring during ripple windows or during intervals between ripple windows, corrected for the different durations of these two types of state. The measure is also required to correct for differences in mean firing rate, because on average the RRUs generated higher rates than NRUs. This measure, termed "relative spike density," was computed as follows for ripple windows and intervals between ripple windows occurring during prebehavioral or postbehavioral rest episodes. First, the spike count was taken for the relevant state and episode under scrutiny (e.g., ripple windows, prebehavioral rest). This spike count was divided by the total spike count occurring in the entire episode under study (e.g., prebehavioral rest). This ratio was next divided by the total period of time made up by the relevant state in the same episode (e.g., total duration of ripple windows during prebehavioral rest).
Within the QW-SWS periods of postbehavioral rest, the mean relative spike density was significantly higher for RRUs during ripple windows than during intervals $\left(4.75 \times 10^{-4} \pm 0.20 \times\right.$ $10^{-4}$ and $2.24 \times 10^{-4} \pm 0.12 \times 10^{-4} \mathrm{~s}^{-1}$, respectively; $p<$ $0.0001 ; n=57)$. In contrast, the relative spike density during prebehavioral rest was similar for ripple windows and intervals $\left(5.91 \times 10^{-4} \pm 0.55 \times 10^{-4}\right.$ and $4.76 \times 10^{-4} \pm 0.12 \times 10^{-4}$ $\mathrm{s}^{-1}$, respectively; NS). The NRUs $(n=166)$, however, showed similar relative spike densities for ripple windows and intervals in both the prebehavioral and postbehavioral rest episodes (prebehavioral rest: ripple windows, $4.93 \times 10^{-4} \pm 0.24 \times 10^{-4}$; intervals, $4.91 \times 10^{-4} \pm 0.12 \times 10^{-4} \mathrm{~s}^{-1}$; NS; postbehavioral rest: ripple windows, $2.57 \times 10^{-4} \pm 0.14 \times 10^{-4}$; intervals, $2.44 \times$ $10^{-4} \pm 0.07 \times 10^{-4} \mathrm{~s}^{-1}$; NS). Comparing the two cell groups during postbehavioral ripple windows, the relative spike density of RRUs was significantly higher than that of NRUs (MWU test, $p<0.001$ ). During the intervals, however, an opposite tendency was expressed, viz. the relative spike density of NRUs was slightly but significantly higher than of RRUs $(p<0.001)$. These differences between RRUs and NRUs were not observed during prebehavioral rest. Thus, not only are reward-related firing patterns more strongly reactivated relative to nonrelated patterns, rewardrelated patterns also become more temporally aligned to ripple episodes during postbehavioral rest, relative to prebehavioral rest and to non-reward-related patterns.

\section{Discussion}

Our main results indicate that reactivation mediated by rewardrelated neurons was significantly stronger compared with neurons not showing such activity and was particularly prominent for spike trains temporally linked to reward sites. A small minority of the units, pairs sharing highly similar firing patterns on the track, accounted for this reactivation, underscoring the sparseness of the phenomenon. Reactivation of reward-related neurons was prevalent during QW-SWS but could not be detected during REM sleep and did not decay across $40 \mathrm{~min}$ of sleep after the task.

Ensemble recordings from rat VS revealed firing patterns that were closely correlated in time to reward site visits. This result agrees with previous studies in rats and primates indicating that VS neurons fire in anticipation of reinforcement and in the postreward phase (Schultz et al., 1992; Roitman et al., 2005). Indeed, no units in our study responded to a particular location on the track that was not directly related to reward. Prearrival responses were marked by upward ramps in firing rate, whereas postarrival responses were usually sensitive to the presence of reward. In agreement with existing literature, the definition of reward-related firing behavior used here is a broad one including sensorimotor as well as valuation aspects of reward approach and consumption. Our first novel findings were that VS reactivation was associated with short time intervals after ripple onset, as previously reported for hippocampus (Kudrimoti et al., 1999). Reactivation was not detected in REM sleep, and this contrast could not be ascribed to a potential undersampling problem for REM sleep or to a temporal arrangement during postbehavioral rest in which REM sleep occurred later than QW-SWS, in combination with a decaying reactivation. Because REM sleep appears important for consolidation of procedural memory (Gais et al., 2000; Stickgold and Walker, 2005), REM replay may occur under different conditions than used here (e.g., in other brain structures than the VS) (Maquet et al., 2000; Louie and Wilson, 2001) or during later sleep stages.

Our most striking observation was that the subgroup of RRUs reactivated very strongly and significantly more so than various 
control groups of NRUs. This difference could not be explained by differences in group size, mean firing rate, or correlation strength. The powerful reactivation of RRUs was accounted for by only a small fraction of highly contributing cell pairs (7.1\%), the members of which showed very similar firing behavior during track running (Fig. 5C). This sparsity offers a parsimonious explanation of the previous (Pennartz et al., 2004) and current observation that reactivation in ventral striatal ensembles, although significant, is highly variable across sessions. Absence of reactivation in individual sessions is likely attributable to a lack of highly correlated cell pairs in the recorded sample. Because the reactivation remained strong when only spikes generated during a narrow time interval linked to reward site arrivals were included in the dataset from the behavioral period, our results indicate that at least reward-related firing patterns are reactivated, whereas other types of information present during the intervals between visits were reprocessed less prominently. What type of motivationally relevant information is reactivated is subject for additional investigation, although the prereward firing of "high contributors" suggests a component of reward expectancy.

The level of reactivation in the rewardrelated subpopulation was consistently higher than in the various nonrelated groups. Therefore, it is tempting to speculate that the motivational value of the information processed during behavior might influence the strength by which it will be reactivated during sleep. Nonetheless, the subpopulation without significant reward-related activity also showed reactivation. The latter effect may have arisen because of reactivation of spike patterns correlated to behavioral components other than reward site visits, or because these cells showed a trend toward being correlated to rewarding events but remained subthreshold for statistical significance. Because NRUs may show a comparably strong reactivation as RRUs in another task setting than described here, additional evidence would be needed to assess a hypothesized coupling between the motivational value represented by neural activity and its reactivation strength.

In contrast to the traditional view of the VS as a primarily "executive" interface that converts limbic, associatively learned information into goal-directed behavior (Mogenson et al., 1980), recent behavioral studies have highlighted its role in learning and memory (Kelley et al., 1997; Parkinson et al., 1999; Corbit et al., 2001), acknowledging the long-term plasticity of intrastriatal glutamatergic afferents (Pennartz et al., 1993; Kombian and Malenka, 1994). Pharmacological studies using postbehavioral intrastriatal injections of protein synthesis inhibitors, NMDA or


Figure 5. Strong reactivation of ventral striatal units showing reward-related firing patterns. $A$, Bootstrap distributions of EV - REV values of reward-related correlates (RRU; blue) and non-reward-related correlates (NRU; green). The RRU distribution was broad and bimodal, indicating inhomogeneous contributions of cell pairs to reactivation. $\boldsymbol{B}$, Distribution of the contributions of individual RRU cell pairs to their session EV. Only a small fraction of the RRU cell pairs contributed $>10 \%$ (5 of 70; high C, Rate maps of two neurons constituting a highly contributing pair. Cross-correlograms show that firing patterns of 列 Reactivation disappeared after exclusion of highly contributing pairs from the total RRU group, as shown by a leftward 列 gher than in control groups matched for recording session (green), firing rate (orange), and strength of Pearson's correlation F EV - REV values plotted as function of the width of the time window before and after reward site visits or reactivated more strongly than those that occurred in intervals (open circles). Reactivation was not observed for the NRU group across the same time windows (green).

dopamine receptor antagonists suggest the VS to contribute to the consolidation of several forms of memory (Setlow and McGaugh, 1998; Hernandez et al., 2002; Sargolini et al., 2003; Dalley et al., 2005). Until recently, a possible neurophysiological mechanism explaining this involvement remained unknown. Given the current evidence that replay comprises a reward-related component, we hypothesize that the VS supports consolidation by endowing reactivation processes with motivational value, although it may also contribute cue- and context-related information.

The empirical support for this thesis may have consequences for our current thinking about memory traces and their off-line replay. Given the evidence that the hippocampus retrieves 
spatial-contextual information by reactivating cells characterized by place fields during active behavior, the VS appears to reactivate a different type of information. Hence it becomes reasonable to assume that both cortical and subcortical brain structures sustain replay in a domain-specific manner (Harris et al., 2001; Ji and Wilson, 2007). Whereas direct sensory processing of object information is generally accepted to occur in many brain areas in parallel and in a domain- or modality-specific way, so too may replay be conceived as a distributed process in which some structures reactivate information about the physical-sensory properties of an object and others about its spatiotemporal context and motivational value. It should be stressed that other brain structures involved in the processing of reward-related information including the medial prefrontal cortex and amygdala may influence or contribute to the reactivation of motivational information in the VS (Paré et al., 2002; Euston et al., 2007).

Regardless of the precise nature of trace storage, reactivation across multiple brain structures such as hippocampus and VS raises the question how pieces of information belonging to the same scene or event are reprocessed coherently, so that erroneous associations with other events can be prevented. Synchronized cross-structural replay (Qin et al., 1997; Hoffman and McNaughton, 2002; Ji and Wilson, 2007) may subserve such a function. As suggested by our data, hippocampal ripples may have a facilitating or coordinating role in supporting this process. Because ventral striatal replay especially occurs during hippocampal ripples and shortly thereafter, these high-frequency oscillations and the associated transitions in neocortical states (Battaglia et al., 2004; Siapas et al., 2005) may act as a release mechanism by which the principal cells of VS reactivate motivational information that will subsequently reach target sites in, for example, ventral pallidum and connected thalamo-prefrontal cortical circuits, lateral hypothalamus and ventral tegmental area (VTA). Recently, Foster and Wilson (2006) reported a reverse replay in hippocampal place cells when rats were pausing at a reward site after running along a track. They suggested that, when this replay coincides with decaying dopamine transients triggered by VTA activity, a reinforcement learning mechanism can be implemented to the effect that reward associations of places close to the reward site are more strongly stored than those of distant places. Such a process would require (1) reverse replay to be temporally aligned with transients in dopamine release and (2) the appropriate reward information to reach the VTA to enable it to produce a presumed teaching signal (Schultz et al., 1997). Hippocampal ripples triggering reactivation of ventral striatal principal neurons projecting to the VTA may provide such a temporal alignment mechanism (Pennartz et al., 2004; Foster and Wilson, 2006). The second requirement may be met by the current evidence for off-line retrieval of value information, which may drive dopaminergic cells to signal errors in reward prediction. In turn, such signals may underlie dopamine-dependent memory consolidation in target structures such as the VS (Dalley et al., 2005). The mechanism by which dopamine release in target structures can be boosted during offline processing is currently unknown. This could be mediated by burst firing of VTA neurons, which however seems predominant during REM sleep but not QW-SWS (Dahan et al., 2007) or enhancement of local dopamine release by excitatory afferent input to target structures (Floresco et al., 2003).

Even if this hippocampal-ventral striatal-VTA loop (Lisman and Grace, 2005) subserves different functions than hypothesized, other VS output pathways, such as the mediodorsal thalamus-medial prefrontal cortex pathway (Zahm and Brog, 1992), are available as potential routes along which motivationally rele- vant information can be supplied to a network of structures involved in the consolidation of reward-dependent learning processes. VS reactivation can be hypothesized to contribute a motivational or emotional component to memory consolidation (McGaugh, 2000; Wagner et al., 2006) not only by strengthening intrastriatal input connections (Pennartz et al., 1993; Kombian and Malenka, 1994) but also by affecting reactivation in other subcortical and cortical networks.

\section{References}

Battaglia FP, Sutherland GR, McNaughton BL (2004) Hippocampal sharp wave bursts coincide with neocortical "up-state" transitions. Learn Mem 11:697-704.

Buzsáki G (1986) Hippocampal sharp waves: their origin and significance. Brain Res 398:242-252.

Corbit LH, Muir JL, Balleine BW (2001) The role of the nucleus accumbens in instrumental conditioning: evidence of a functional dissociation between accumbens core and shell. J Neurosci 21:3251-3260.

Dahan L, Astier B, Vautrelle N, Urbain N, Kocsis B, Chouvet G (2007) Prominent burst firing of dopaminergic neurons in the ventral tegmental area during paradoxical sleep. Neuropsychopharmacology 32:1232-1241.

Dalley JW, Lääne K, Theobald DE, Armstrong HC, Corlett PR, Chudasama Y, Robbins TW (2005) Time-limited modulation of appetitive Pavlovian memory by D1 and NMDA receptors in the nucleus accumbens. Proc Natl Acad Sci USA 102:6189-6194.

Euston DR, Tatsuno M, McNaughton BL (2007) Fast-forward playback of recent memory sequences in prefrontal cortex during sleep. Science 318:1147-1150.

Floresco SB, West AR, Ash B, Moore H, Grace AA (2003) Afferent modulation of dopamine neuron firing differentially regulates tonic and phasic dopamine transmission. Nat Neurosci 6:968-973.

Foster DJ, Wilson MA (2006) Reverse replay of behavioural sequences in hippocampal place cells during the awake state. Nature 440:680-683.

Gais S, Plihal W, Wagner U, Born J (2000) Early sleep triggers memory for early visual discrimination skills. Nat Neurosci 3:1335-1339.

Harris JA, Harris IM, Diamond ME (2001) The topography of tactile learning in humans. J Neurosci 21:1056-1061.

Hassani OK, Cromwell HC, Schultz W (2001) Influence of expectation of different rewards on behavior-related neuronal activity in the striatum. J Neurophysiol 85:2477-2489.

Hernandez PJ, Sadeghian K, Kelley AE (2002) Early consolidation of instrumental learning requires protein synthesis in the nucleus accumbens. Nat Neurosci 5:1327-1331.

Hoffman KL, McNaughton BL (2002) Coordinated reactivation of distributed memory traces in primate neocortex. Science 297:2070-2073.

Jackson JC, Johnson A, Redish AD (2006) Hippocampal sharp waves and reactivation during awake states depend on repeated sequential experience. J Neurosci 26:12415-12426.

Ji D, Wilson MA (2007) Coordinated memory replay in the visual cortex and hippocampus during sleep. Nat Neurosci 10:100-107.

Kelley AE, Smith-Roe SL, Holahan MR (1997) Response-reinforcement learning is dependent on $N$-methyl-D-aspartate receptor activation in the nucleus accumbens core. Proc Natl Acad Sci USA 94:12174-12179.

Kleinbaum DG, Kupper LL, Muller KE, Nizam A (1998) Applied regression analysis and other multivariate methods. Boston: Duxbury.

Kombian SB, Malenka RC (1994) Simultaneous LTP of non-NMDA- and LTD of NMDA-receptor-mediated responses in the nucleus accumbens. Nature 368:242-246.

Kudrimoti HS, Barnes CA, McNaughton BL (1999) Reactivation of hippocampal cell assemblies: effects of behavioral state, experience, and EEG dynamics. J Neurosci 19:4090-4101.

Lavoie AM, Mizumori SJ (1994) Spatial, movement- and reward-sensitive discharge by medial ventral striatum neurons of rats. Brain Res 638:157-168.

Lisman JE, Grace AA (2005) The hippocampal-VTA loop: controlling the entry of information into long-term memory. Neuron 46:703-713.

Louie K, Wilson MA (2001) Temporally structured replay of awake hippocampal ensemble activity during rapid eye movement sleep. Neuron 29:145-156.

Maquet P, Laureys S, Peigneux P, Fuchs S, Petiau C, Phillips C, Aerts J, Del Fiore G, Degueldre C, Meulemans T, Luxen A, Franck G, Van Der Linden 
M, Smith C, Cleeremans A (2000) Experience-dependent changes in cerebral activation during human REM sleep. Nat Neurosci 3:831-836.

McGaugh JL (2000) Memory-a century of consolidation. Science 287:248-251.

McNaughton BL (1998) The neurophysiology of reminiscence. Neurobiol Learn Mem 70:252-267.

Mogenson GJ, Jones DL, Yim CY (1980) From motivation to action: functional interface between the limbic system and the motor system. Prog Neurobiol 14:69-97.

O’Doherty JP, Buchanan TW, Seymour B, Dolan RJ (2006) Predictive neural coding of reward preference involves dissociable responses in human ventral midbrain and ventral striatum. Neuron 49:157-166.

O'Keefe J, Nadel L (1978) The hippocampus as a cognitive map: Oxford: Clarendon.

Paré D, Collins DR, Pelletier JG (2002) Amygdala oscillations and the consolidation of emotional memories. Trends Cogn Sci 6:306-314.

Parkinson JA, Olmstead MC, Burns LH, Robbins TW, Everitt BJ (1999) Dissociation in effects of lesions of the nucleus accumbens core and shell on appetitive pavlovian approach behavior and the potentiation of conditioned reinforcement and locomotor activity by $\mathrm{D}$-amphetamine. J Neurosci 19:2401-2411.

Pavlides C, Winson J (1989) Influences of hippocampal place cell firing in the awake state on the activity of these cells during subsequent sleep episodes. J Neurosci 9:2907-2918.

Paxinos G, Watson C (1996) The rat brain in stereotactic coordinates. New York: Academic.

Pennartz CM, Ameerun RF, Groenewegen HJ, Lopes da Silva FH (1993) Synaptic plasticity in an in vitro slice preparation of the rat nucleus accumbens. Eur J Neurosci 5:107-117.

Pennartz CM, Lee E, Verheul J, Lipa P, Barnes CA, McNaughton BL (2004) The ventral striatum in off-line processing: ensemble reactivation during sleep and modulation by hippocampal ripples. J Neurosci 24:6446-6456.

Qin YL, McNaughton BL, Skaggs WE, Barnes CA (1997) Memory reprocessing in corticocortical and hippocampocortical neuronal ensembles. Philos Trans R Soc Lond B Biol Sci 352:1525-1533.

Rasch B, Büchel C, Gais S, Born J (2007) Odor cues during slow-wave sleep prompt declarative memory consolidation. Science 315:1426-1429.

Robbins TW, Everitt BJ (1996) Neurobehavioural mechanisms of reward and motivation. Curr Opin Neurobiol 6:228-236.

Roitman MF, Wheeler RA, Carelli RM (2005) Nucleus accumbens neurons are innately tuned for rewarding and aversive taste stimuli, encode their predictors, and are linked to motor output. Neuron 45:587-597.

Sargolini F, Florian C, Oliverio A, Mele A, Roullet P (2003) Differential involvement of NMDA and AMPA receptors within the nucleus accum- bens in consolidation of information necessary for place navigation and guidance strategy of mice. Learn Mem 10:285-292.

Schultz W, Apicella P, Scarnati E, Ljungberg T (1992) Neuronal activity in monkey ventral striatum related to the expectation of reward. J Neurosci 12:4595-4610.

Schultz W, Dayan P, Montague PR (1997) A neural substrate of prediction and reward. Science 275:1593-1599.

Setlow B, McGaugh JL (1998) Sulpiride infused into the nucleus accumbens posttraining impairs memory of spatial water maze training. Behav Neurosci 112:603-610.

Setlow B, Schoenbaum G, Gallagher M (2003) Neural encoding in ventral striatum during olfactory discrimination learning. Neuron 38:625-636.

Shibata R, Mulder AB, Trullier O, Wiener SI (2001) Position sensitivity in phasically discharging nucleus accumbens neurons of rats alternating between tasks requiring complementary types of spatial cues. Neuroscience 108:391-411.

Shidara M, Aigner TG, Richmond BJ (1998) Neuronal signals in the monkey ventral striatum related to progress through a predictable series of trials. J Neurosci 18:2613-2625.

Siapas AG, Lubenov EV, Wilson MA (2005) Prefrontal phase locking to hippocampal theta oscillations. Neuron 46:141-151.

Skaggs WE, McNaughton BL (1996) Replay of neuronal firing sequences in rat hippocampus during sleep following spatial experience. Science 271:1870-1873.

Sokal RR, Rohlf FJ (1995) Biometry, pp 823-825. New York: Freedman.

Stickgold R, Walker MP (2005) Memory consolidation and reconsolidation: what is the role of sleep? Trends Neurosci 28:408-415.

Tatsuno M, Lipa P, McNaughton BL (2006) Methodological considerations on the use of template matching to study long-lasting memory trace replay. J Neurosci 26:10727-10742.

Tran AH, Tamura R, Uwano T, Kobayashi T, Katsuki M, Ono T (2005) Dopamine D1 receptors involved in locomotor activity and accumbens neural responses to prediction of reward associated with place. Proc Natl Acad Sci USA 102:2117-2122.

Tremblay L, Hollerman JR, Schultz W (1998) Modifications of reward expectation-related neuronal activity during learning in primate striatum. J Neurophysiol 80:964-977.

Vanderwolf CH (1969) Hippocampal electrical activity and voluntary movement in the rat. Electroencephalogr Clin Neurophysiol 26:407-418.

Wagner U, Hallschmid M, Rasch B, Born J (2006) Brief sleep after learning keeps emotional memories alive for years. Biol Psychiatry 60:788-790.

Wilson MA, McNaughton BL (1994) Reactivation of hippocampal ensemble memories during sleep. Science 265:676-679.

Zahm DS, Brog JS (1992) On the significance of subterritories in the "accumbens" part of the rat ventral striatum. Neuroscience 50:751-767. 